\title{
Isolas of periodic passive Q-switching self-pulsations in the three-level:two-level model for a laser with a saturable absorber
}

\author{
Eusebius J. Doedel ${ }^{1}$ and Carlos L. Pando L. ${ }^{2}$ \\ ${ }^{1}$ Department of Computer Science, Concordia University, 1455 boulevard de Maisonneuve O., Montréal, Québec H3G 1M8, Canada \\ ${ }^{2}$ Instituto de Física, Benemérita Universidad Autónoma de Puebla, Apartado Postal J-48, Puebla, Pue. 72570, Mexico
}

(Received 5 January 2011; revised manuscript received 7 July 2011; published 7 November 2011)

\begin{abstract}
We show that a fundamental feature of the three-level:two-level model, used to describe molecular monomode lasers with a saturable absorber, is the existence of isolas of periodic passive Q-switching (PQS) self-pulsations. A common feature of these closed families of periodic solutions is that they contain regions of stability of the PQS self-pulsation bordered by period-doubling and fold bifurcations, when the control parameter is either the incoherent external pump or the cavity frequency detuning. These findings unveil the fundamental solution structure that is at the origin of the phenomenon known as "period-adding cascades" in our system. Using numerical continuation techniques we determine these isolas systematically, as well as the changes they undergo as secondary parameters are varied.
\end{abstract}

DOI: 10.1103/PhysRevE.84.056207

PACS number(s): 05.45.-a, 42.60.Gd, 42.65.Sf

\section{INTRODUCTION}

Single-mode class-B lasers, where polarization dynamics is adiabatically eliminated, typically do not show dynamical instabilities unless additional electro-optical or optical components, such as intracavity modulators, saturable absorbers, external field injections, or external feedbacks, are introduced in the laser system [1-3]. An important example of these lasers, the $\mathrm{CO}_{2}$ laser with an intracavity gaseous saturable absorber (LSA), may generate giant laser spikes, which are known as passive Q-switching (PQS) self-pulsations. The latter were first observed a long time ago in the laboratory by Wood and Schwarz in 1967 [4]. Soon after, the complex dynamics induced by the $\mathrm{CO}_{2}$ LSA experiments triggered a number of early studies [2,5-9]. Indeed, the rich dynamical phenomenology of the $\mathrm{CO}_{2} \mathrm{LSA}$, and its versatility to change control parameters, make it a particularly interesting object for the study of its nonlinear dynamics [10-16].

In this paper we show that a widely accepted model, the three-level:two-level model (3-2LM), which describes the temporal dynamics of this system with a good degree of fidelity $[10,16]$, predicts the existence of isolas of periodic PQS self-pulsations as a suitable parameter is changed. This parameter can be the incoherent external pump or the cavity frequency detuning. By an isola, we mean a smooth, closed, and isolated family of periodic solutions, stable or unstable, which is generated as a single control parameter is allowed to change. Isolas of periodic orbits in autonomous systems have been found in different contexts, such as in an electronic circuit [17], and in a model for air oxidation of hydrogen in a continuous-flow stirred tank reactor [18,19].

In the $\mathrm{CO}_{2} \mathrm{LSA}$, typically each PQS self-pulsation consists of a leading spike, named the reinjection, and $n-1$ small intensity undulations in a tail $[9,10]$. We label these periodic PQS self-pulsations as $\Pi^{(n)}$. As the incoherent external pump $[13,20]$ or the cavity frequency detuning $[16]$ is changed in the experiment, period-adding cascades of PQS self-pulsations arise, $\Pi^{(n)} \rightarrow \Pi^{(n+1)}$ or vice versa. In the framework of the three-level:two-level model (3-2LM) $[9,10,13]$, we show that, as the incoherent pump or the cavity frequency detuning is changed, the origin of the period-adding cascades stems from the peculiar coexistence of stable $\Pi^{(n)}$ along a sequence of isolas of periodic orbits of increasingly higher period for increasing $n$.

Previous studies on period-adding cascades have been reported for optically injected lasers [21-24], and for a laser rate equation model with a term describing a saturable absorber and a modulated pump source [25]. To our knowledge, however, no previous observation of isolas of $\mathrm{PQS}$ self-pulsations has been reported in PQS semiconductor lasers [26] or in PQS microchip lasers [27].

\section{THREE-LEVEL:TWO-LEVEL MODEL}

The standard model for the $\mathrm{CO}_{2} \mathrm{LSA}$ is described by four coupled rate equations for the laser intensity $I$, the population densities in the upper and lower laser levels of the amplifier, and the population density difference between the energy levels in the absorber $[9,10]$. In this model it is assumed that both the amplifier lasing molecular transition frequency and that of the absorber are homogeneously broadened and resonant. It has also been shown that the 3-2LM describes the $\mathrm{N}_{2} \mathrm{O}$ LSA experimental results [20]. After a suitable normalization of the variables and parameters, the following set of equations is obtained $[13,16]$ :

$$
\begin{aligned}
\frac{d I}{d t} & =I(U-\bar{U}-1), \quad \frac{d U}{d t}=\epsilon[W-U(1+I)], \\
\frac{d W}{d t} & =\epsilon(A+b U-W), \quad \frac{d \bar{U}}{d t}=\bar{\epsilon}[\bar{A}-\bar{U}(1+a I)] .
\end{aligned}
$$

Here $U$ and $W$ are proportional to the active population difference and the effective population source term, respectively, in the amplifier, $\bar{U}$ is proportional to the population difference in the absorber, and $\epsilon$ and $\bar{\epsilon}$ are normalized relaxation rates in the amplifier and absorber, respectively. The parameter $a$ is proportional to the ratio of the absorption coefficient to the amplification coefficient. Finally, $b$ is the squared ratio between two effective relaxation rates in the amplifier. The parameter $A$ is proportional to the external incoherent pump rate in the gain medium, while $\bar{A}$ is proportional to the equilibrium population difference in the absorber. The time 
$t$ has been rescaled with respect to the cavity-damping rate. In the case when the cavity frequency detuning $\Delta \omega$ is the main control parameter, a theoretical dependence of the laser parameters $A, \bar{A}$, and $a$ on $\Delta \omega$ has been proposed [16], to be discussed later in connection with Eq. (2).

When $A$ is the main control parameter the numerical values assigned to the parameters in Eq. (1) are the following [13,16]: $\epsilon=0.137, \bar{\epsilon}=8.00, a=4.17, b=0.85$, and $\bar{A}=3.50$. These values indicate that there is a fast variable, $I$, and two slow variables, $U$ and $W$. The variable $\bar{U}$ is seen to be a very fast variable and typically it is eliminated from Eq. (1) via a quasisteady state approximation to simplify the study. Furthermore, time integration of Eq. (1) suggests that the dynamics switches between slow and fast motion and small and large amplitudes. Systems with these features are known as mixed mode oscillations (MMOs) and are the subject of substantial current research [28]. MMOs are ubiquitous in nature and have been observed in diverse systems such as in surface chemical reactions, electrochemical systems, and neural systems, to name just a few fields [28].

(a)

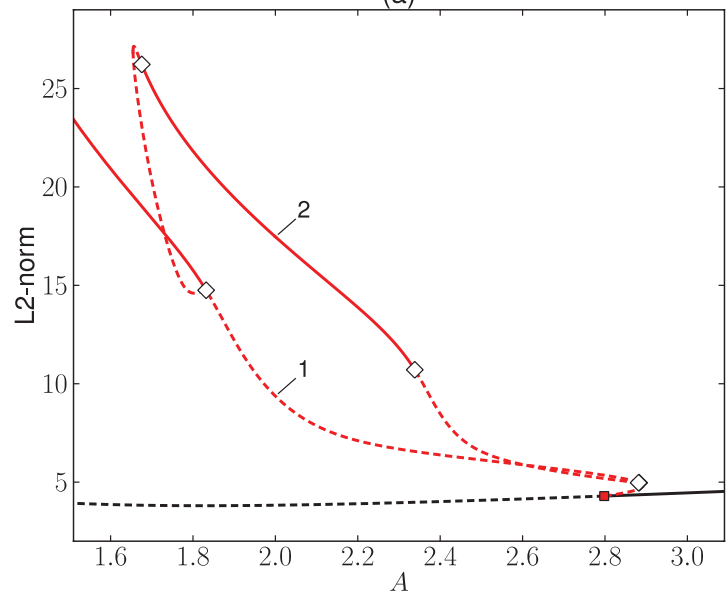

(c)

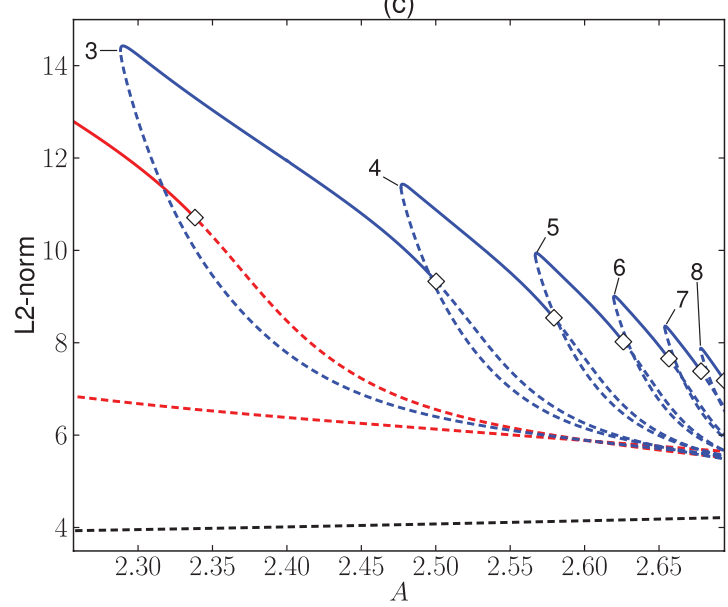

The basic stationary solution

$$
I=0, \quad U=W=\frac{A}{1-b}, \quad \bar{U}=\bar{A},
$$

is stable when $A<(1-b)(1+\bar{A})$. It loses stability at $A=$ $(1-b)(1+\bar{A})$ as a result of a transcritical bifurcation where the basic solution family and a secondary stationary solution family intersect. Thus for our parameter values the basic solution loses stability at $A=0.675$. One leg of the bifurcating family of secondary stationary solutions $(I>0)$ consists of unstable solutions until a Hopf bifurcation is reached at $A \approx 2.8$, beyond which these equilibria become stable. The latter can be seen in the basic bifurcation diagram in Fig. 1(a).

The numerical computations, carried out with AUTO [29], sensitively depend on how small $I$ gets. For this reason, it is better to use a new variable $c$, where $I=e^{c}$. The basic solution, $I=0$, then corresponds to $c=-\infty$. Due to this transformation the basic solution cannot be seen in the bifurcation diagrams, most of which show the $L_{2}$ norm versus

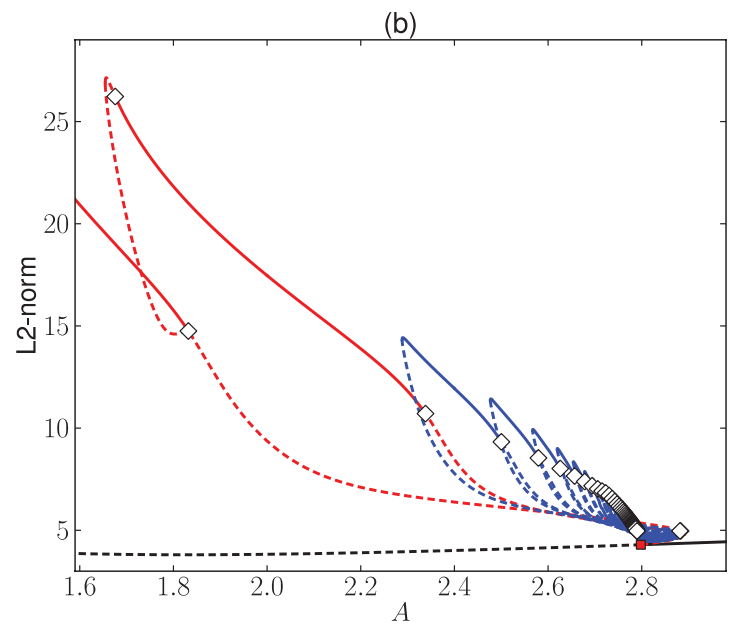

(d)

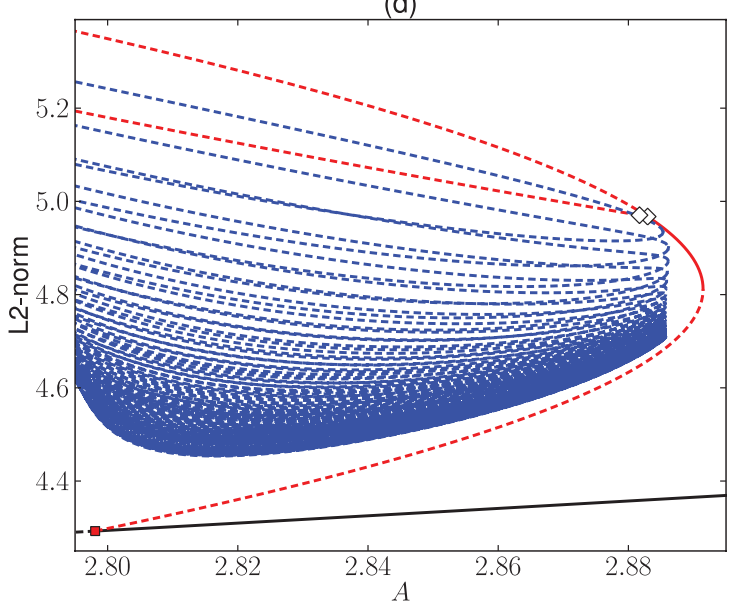

FIG. 1. (Color online) (a) The bifurcation diagram, with norm versus $A$, showing the stationary family with Hopf bifurcation (small square), the one-pulse family $\Pi^{(1)}$ (labeled 1), and a period-doubled family $\Pi^{(2)}$ of two-pulse orbits (labeled 2), which connects two period-doubling bifurcations (open diamonds) on the one-pulse family. (b) The bifurcation diagram, also showing the isolas $\mathcal{I}_{n}, n=3,4, \ldots, 50$. (c) A detail of (b), showing part of isolas $\mathcal{I}_{3}-\mathcal{I}_{8}$ (labeled 3-8). (d) A further detail of (b), showing part of the isolas $\mathcal{I}_{n} n=3,4, \ldots, 50$, close to their right-hand folds near $A=2.88$. Not indicated are period doublings that are extremely close to these folds. 
$A$. For stationary solutions $(I>0)$, this norm is

$$
\sqrt{c^{2}+U^{2}+W^{2}+\bar{U}^{2}}
$$

while for periodic solutions of period $T$ we use

$$
\frac{1}{\sqrt{T}}\left[\int_{0}^{T} c(t)^{2}+U(t)^{2}+W(t)^{2}+\bar{U}_{2}(t)^{2} d t\right]^{1 / 2} .
$$

We note that bifurcation diagrams are intended to give a schematic representation of the bifurcation behavior of a system. Towards this purpose the choice of the vertical axis is not so important, the main criterion for its choice being the clarity of the diagram. Although the $L_{2}$ norm, as defined above, may not be of physical interest in the current context, it was found to give diagrams that are quite clear compared to other choices.

\section{ISOLAS OF PERIODIC PQS SELF-PULSATIONS}

In the framework of the 3-2LM we show that isolas of PQS periodic self-pulsations, $\Pi^{(n)}$, arise when either the incoherent pump parameter $A$ in the amplifier or the cavity frequency detuning, $\Delta \omega$, is changed. The former case is considered in detail, while the latter is briefly mentioned as any qualitative information about it can be obtained from the former case. We discuss both physical situations because period-adding cascades of $\Pi^{(n)}$ arise when either $A[13,20]$ or $\Delta \omega[16]$ is changed in an experiment. We explain the origin of these cascades as being the result of the peculiar coexistence of stable intervals for $\Pi^{(n)}$ along different isolas.

The basic bifurcation diagram displayed in Fig. 1(a) also shows the family of periodic solutions $\Pi^{(1)}$ that emanates from the Hopf bifurcation at $A \approx 2.8$ as the incoherent pump parameter $A$ changes. Note that this family contains a fold, as well as two period-doubling bifurcations, namely, near $A \approx 1.83$ and $A \approx 2.88$, of which the latter is near the fold. The periodic solutions are stable to the left of the perioddoubling bifurcation near $A \approx 1.83$. Figure 1 (a) also shows that the two period-doubling bifurcations are connected by a

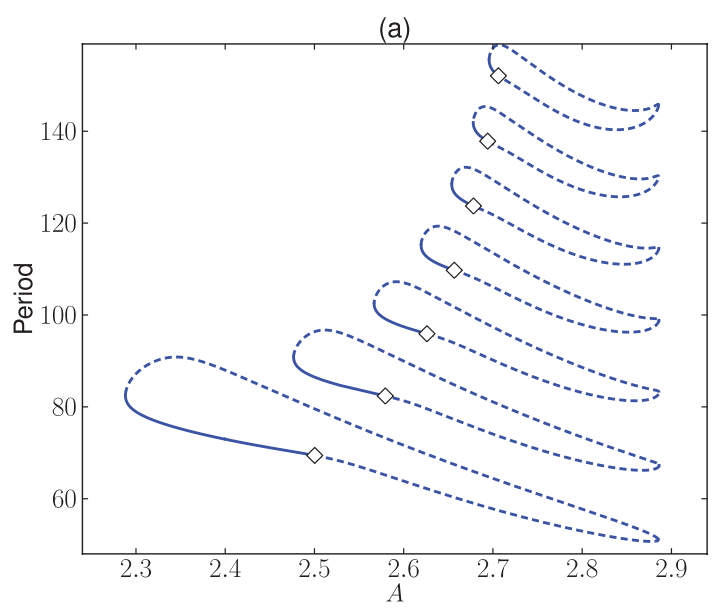

single period-doubled family $\Pi^{(2)}$, which contains a region of stability that is bordered by secondary period-doubling bifurcations, namely, near $A \approx 1.67$ and $A \approx 2.39$. These secondary period-doubling bifurcations can lead to more complicated stable dynamics that, as we have found, is mostly confined to very small intervals in parameter space. We note that periodic orbits reported in this paper are computed by boundary-value algorithms, as implemented in AUTO [29].

The origin of the period-adding cascades stems from the coexistence of the stable periodic PQS self-pulsations $\Pi^{(n)}$, and for this reason we consider the organization of their stability regions. To this end, first note that $\Pi^{(2)}$ in Fig. 1(a) corresponds to the period-doubled orbit, which emanates from the main periodic PQS self-pulsation $\Pi^{(1)}$.

Figure 1(b) shows that there are additional periodic solution families $\Pi^{(n)}$ that cannot be reached directly by periodic solution bifurcations from the families found in Fig. 1(a), at least not by only varying the parameter $A$. Specifically, Fig. 1(b) shows a number of isolated families (isolas) of periodic solutions. We shall refer to these isolas as $\mathcal{I}_{n}$, $n=3,4,5,6, \ldots$ Enlarged views of Fig. 1(b) are shown in Figs. 1(c) and 1(d).

In Fig. 2(a) the isolas $\mathcal{I}_{3}, \ldots, \mathcal{I}_{9}$ are shown with the period of their orbits as the vertical axis. It is this diagram that most convincingly displays the isolated nature of the solution families $\mathcal{I}_{n}$. Figure 2(b) also shows these isolas, but with the maximum of the solution component $c(t)$ as vertical axis.

The periodic self-pulsation $\Pi^{(n)}$ belongs to the isola $\mathcal{I}_{n}, n=$ $3,4,5,6, \ldots$ Figure 2 (a) shows that each isola $\mathcal{I}_{n}$ contains fold and period-doubling bifurcations. On each isola the periodic self-pulsations $\Pi^{(n)}$ are stable in an $A$ interval bounded on the left by a fold, and on the right by a period-doubling bifurcation, as most clearly seen in Figs. 1(c) and 2(a). In the figures we can observe that stable self-pulsations $\Pi^{(n)}$, for different $n$, coexist before the Hopf bifurcation at $A \approx 2.8$; see, e.g., Figs. 1(b) and 2(a). When the incoherent pump $A$ is changed, this peculiar coexistence of periodic attractors $\Pi^{(n)}$ is the reason for the origin of systematic hysteresis jumps observed experimentally $[13,20]$, namely, the period-adding cascades.

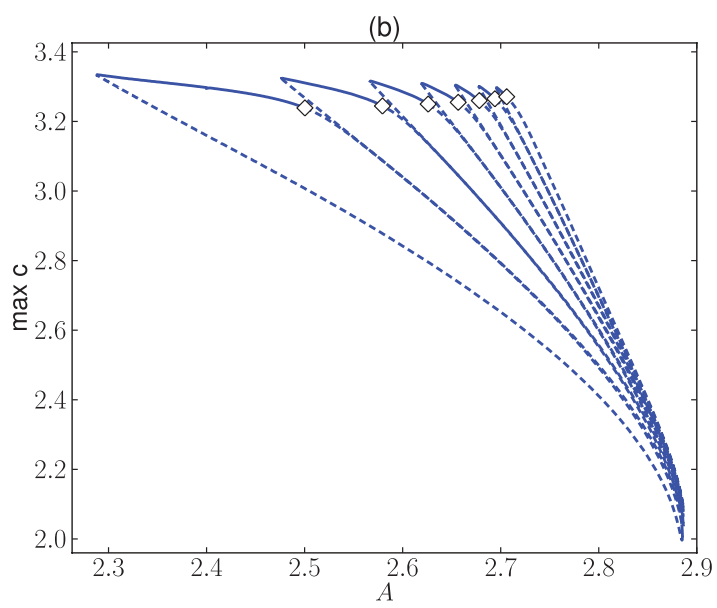

FIG. 2. (Color online) The isolas $\mathcal{I}_{3}, \ldots, \mathcal{I}_{9}$, showing (a) period versus $A$, and (b) max $c$ versus $A$. Open diamonds represent period-doubling bifurcations. Solid/dashed curves represent stable/unstable solutions. Not indicated are period doublings that are extremely close to the right-hand folds. Note that the periods of the orbits along the isola $\mathcal{I}_{n}$ are smaller than those along the isola $\mathcal{I}_{n+1}$; instead the intervals of stability along the isola $\mathcal{I}_{n}$ are larger than those along the isola $\mathcal{I}_{n+1}$. 


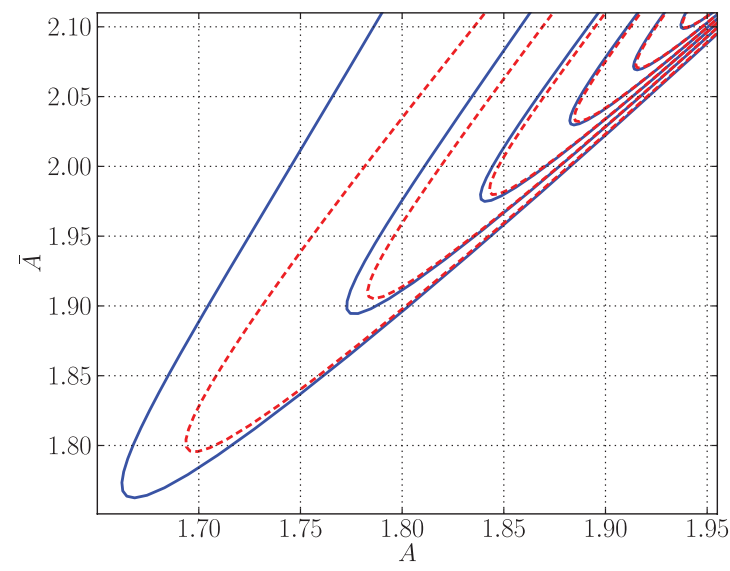

FIG. 3. (Color online) Loci of period-doubling bifurcations (dashed) and loci of folds (solid) for isolas $\mathcal{I}_{3}-\mathcal{I}_{8}$. The minimum of the solid line corresponding to isola $\mathcal{I}_{n}$ is smaller than that for isola $\mathcal{I}_{n+1}$. The same argument holds for the dashed lines.

For higher values of $n$ the stability regions along the isolas $\mathcal{I}_{n}$, delimited by a period-doubling bifurcations and a fold, tend to contract, as seen in Figs. 1 and 2. This contraction can also be seen in Fig. 3, which shows the loci of period doublings and folds in the two parameters $A$ and $\bar{A}$. Figure 3 also shows that the isolas themselves shrink and disappear one by one as $\bar{A}$ decreases.

Extremely small regions of stability along the isolas $\mathcal{I}_{n}$ also occur near the right-hand folds of the isolas, of which a blowup is shown in Fig. 1(d). More precisely, there are period doublings very close to these right-hand folds, which AUTO fails to detect, as they are so close to the fold that a real multiplier moves into the unit circle through +1 (the fold), interacts with a multiplier that is already inside the unit circle (becoming complex, and "circling" around the origin), and leaves the unit circle through -1 (the period doubling), or conversely. All this occurs "in one continuation step." The existence of these right-hand period doublings is best verified by two-parameter continuation, as shown in Fig. 3. However, since the stable region between these period doublings and the right-hand folds is typically very small, we consider them to be less physically relevant. Along each isola we also found that the family that bifurcates from the indicated period-doubling bifurcation in, for example, Fig. 2(a), connects directly to the period-doubling bifurcation (not shown) near the right-hand fold of the isola. These families contain even smaller regions of stability, which we also choose not to describe in detail here.

Near the right-hand folds of the isolas the periodic PQS self-pulsations $\Pi^{(n)}$ with a large number of undulations, i.e., for large $n$, appear to approximate a homoclinic connection from a basic periodic orbit $\Pi^{(1)}$ to itself. At the folds these periodic orbits, again for large $n$, then correspond to approximate homoclinic tangencies. The existence of homoclinic tangencies has been supported both by experimental investigation of the time series in the $\mathrm{CO}_{2}$ LSA $[13,16,30]$ and theoretical studies of the 3-2LM [13,16,31,32]. More precisely, the association of the flow dynamics of Eq. (1) or the experimental time series with suitable multibranched maps as a secure identification method for the presence of homoclinic tangencies has been

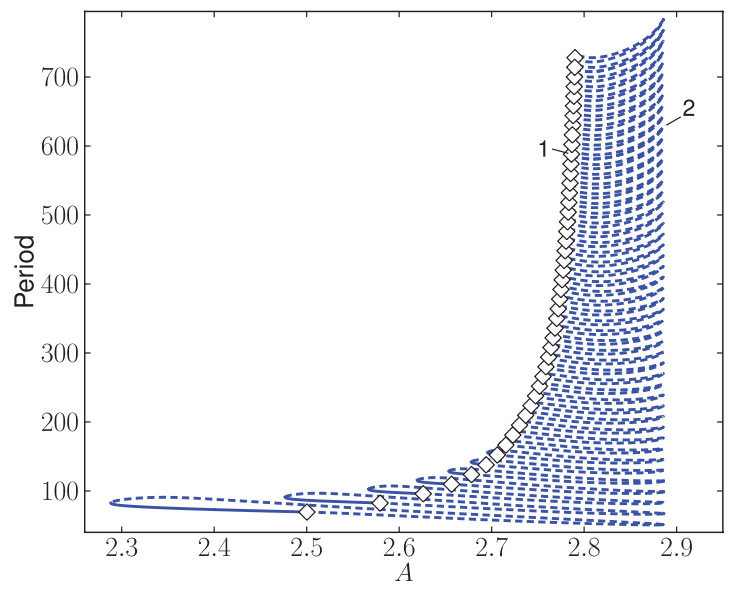

FIG. 4. (Color online) The isolas $\mathcal{I}_{3}, \ldots, \mathcal{I}_{50}$, showing period versus $A$. Open diamonds represent period-doubling bifurcations. Solid/dashed curves represent stable/unstable solutions. Not indicated are period doublings that are extremely close to the right-hand folds. The labels 1 and 2 on isola $\mathcal{I}_{40}$ point at the period-doubling bifurcation and at the right-hand fold, respectively. For a comparison see Fig. 2(a).

considered in several studies [13,16,30,33]. As a manifestation of the possible presence of a homoclinic tangency, Figs. 2(a) and 4 show that the period of the orbits along the isola $\mathcal{I}_{n}$ increases as $n$ increases. This holds in particular for the periodic solutions at the right-hand folds of the isolas. The fact that these solutions approximate a homoclinic orbit, and hence a homoclinic tangency, is illustrated for $\mathcal{I}_{40}$ in Figs. 5(b) and 5(c). Specifically, solution 2 at the right-hand fold of $\mathcal{I}_{40}$ is approximately homoclinic to a periodic solution at $A \approx 2.8857$ along the lower part of the basic family $\Pi^{(1)}$ that emanates from the Hopf bifurcation in Fig. 1(a).

Figure 4 also shows that as $n$ becomes larger the $A$ values of the folds that delimit the isolas on the right approach a limit, namely the value where the homoclinic tangency occurs. Figure 5(b) shows the sinusoidal shape of the undulations of solution 2. In fact, these observations are also at the heart of our procedure to construct starting solutions along isola $\mathcal{I}_{n}$ for larger values of $n$. More precisely, splicing in an additional undulation in a solution near the right-hand fold of $\Pi^{(n)}$ provides an approximate starting solution near the right-hand fold of $\Pi^{(n+1)}$, which is then refined by Newton iteration, and subsequently continued in order to trace out the entire isola $\mathcal{I}_{n}$.

In general, the origin of isolas has been associated to resonance zones close to the tip where the Arnold tongues emerge [34], to cusp bifurcations and Shilnikov homoclinic connections [17], to a neighborhood of a homoclinic tangency to a periodic orbit [35], and to a neighborhood of a ShilnikovHopf bifurcation [36]. In particular, the latter paper contains bifurcation diagrams with isolas that resemble those found in our model. However, in the parameter regime that we consider, we have not yet encountered a Shilnikov-Hopf point. We do find that, as the secondary parameter $\bar{A}$ is decreased and the isolas shrink and sequentially disappear (see Fig. 3), the bifurcation scenario changes to a period-doubling route in the form of a period-doubling bubble, such as in the case of the $\mathrm{CO}_{2}$ with modulated cavity losses, whose frequency is 

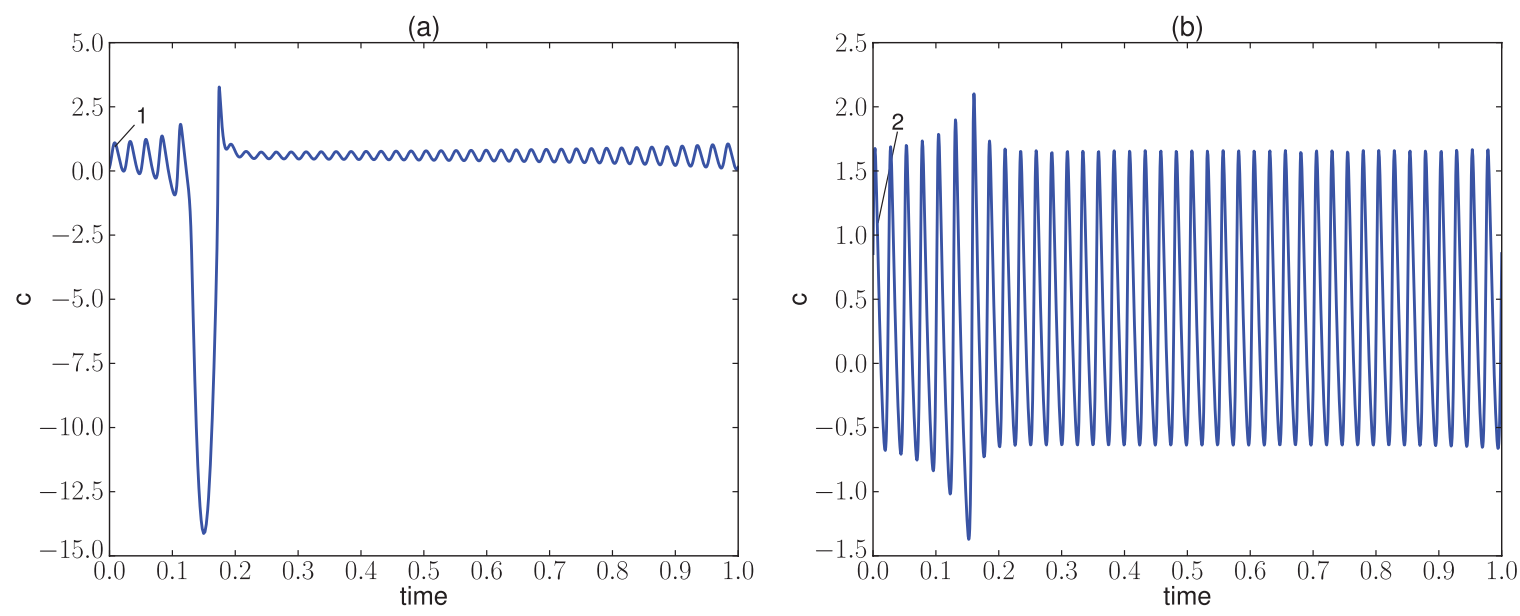

(c)

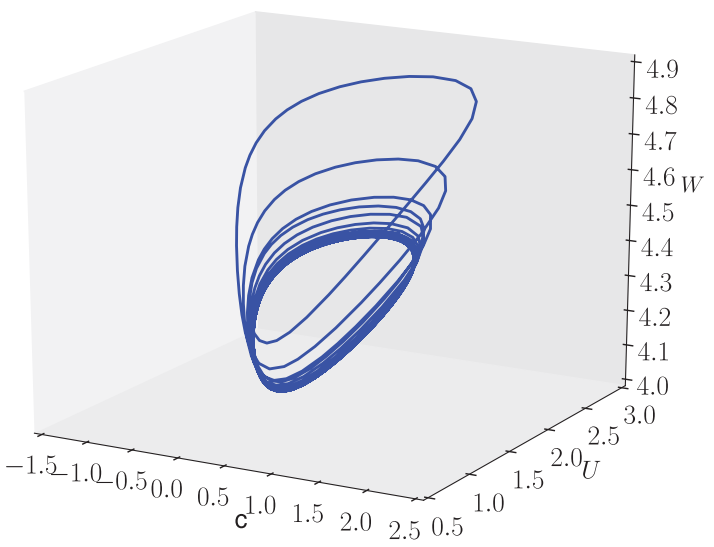

FIG. 5. (Color online) Two periodic solutions along the isola $\mathcal{I}_{40}$, for $\epsilon=0.137, \bar{\epsilon}=8.00, a=4.17, b=0.85$, and $\bar{A}=3.50$. Each $\mathrm{PQS}$ self-pulsation along $\mathcal{I}_{40}$ has a leading spike (the reinjection), and 39 undulations (relaxations). (a) Solution 1 at the period-doubling bifurcation at $A=2.786$. Time has been scaled to the unit interval; the actual period is 588.0. The large negative value of $c$, down to less than -15 , corresponds to a very small value of $I$, where $I=e^{c}$. (b) Solution 2 at the right-hand fold at $A=2.885$, where the sinusoidal undulations are apparent. Time has been scaled; the actual period is 627.6. (c) A three-dimensional representation of solution 2.

changed [37]. A more detailed two-parameter numerical study of this transition would be of mathematical interest in future work.

The presence of a homoclinic orbit biasymptotic to an unstable periodic orbit has also been found in the context of the $\mathrm{CO}_{2}$ LSA in experimental studies [13,16,30], as well as in simulations with different LSA models $[13,16,31,32]$. These models are compatible with the topological organization of the periodic orbits extracted from the experimental chaotic time series [38].

In the present paper, however, our focus is on the study of invariant objects in phase space and their bifurcations, a description that may help to better understand the dynamics of Eq. (1) in its full complexity. To support our findings, we have recently also studied [39] an alternative model for the $\mathrm{CO}_{2}$ LSA [40] for the case of a fast saturable absorber [39]. In this model a bifurcation structure with features qualitatively similar to those discussed in the current paper has been found as the incoherent pump parameter changes [39].

In order to compare our findings with those of experiments $[9,10,13,14,20]$, we also numerically integrated Eq. (1). In Fig. 6, as $A$ is decreased, a period-adding cascade, $\Pi^{(n+1)} \rightarrow$
$\Pi^{(n)}$ with $n=3,4,5,6, \ldots$, is visible. The cascades are evident by comparing Fig. 6 to, for example, Figs. 1(c) and 2(a), with the understanding that $A$ is being decreased. Note that in Fig. 6, just before the onset of chaotic behavior near $A=2.8$, the system is in its stable equilibrium. The stability of the secondary stationary solution is lost as a result of the Hopf bifurcation at $A \approx 2.8$, as is evident by comparing Fig. 6 and, for example, Fig. 1(a). The periodic time evolution of the PQS self-pulsations, $\Pi^{(n)}$, is shown in Fig. 7 for $n=3,4,5,6$, each of which arises for a different value of $A$.

We also have considered the bifurcations that the $\mathrm{CO}_{2} \mathrm{LSA}$ system undergoes as the cavity frequency detuning changes. Within the framework of the 3-2LM in Eq. (1), a theoretical dependence of the laser parameters $A, \bar{A}$, and $a$ on the cavity frequency detuning relative to that of the atomic frequency, $\Delta \omega$, has been suggested [16,41]. In this model the lasing transition frequencies of both the homogeneously broadened amplifier and absorber are the same, and the laser frequency is assumed to be that of the optical cavity. For suitable parameter values a qualitative agreement between the results of this model and those of the experiment has been shown [16]. Within this framework [16] we have found that as $\Delta \omega$ changes and for 


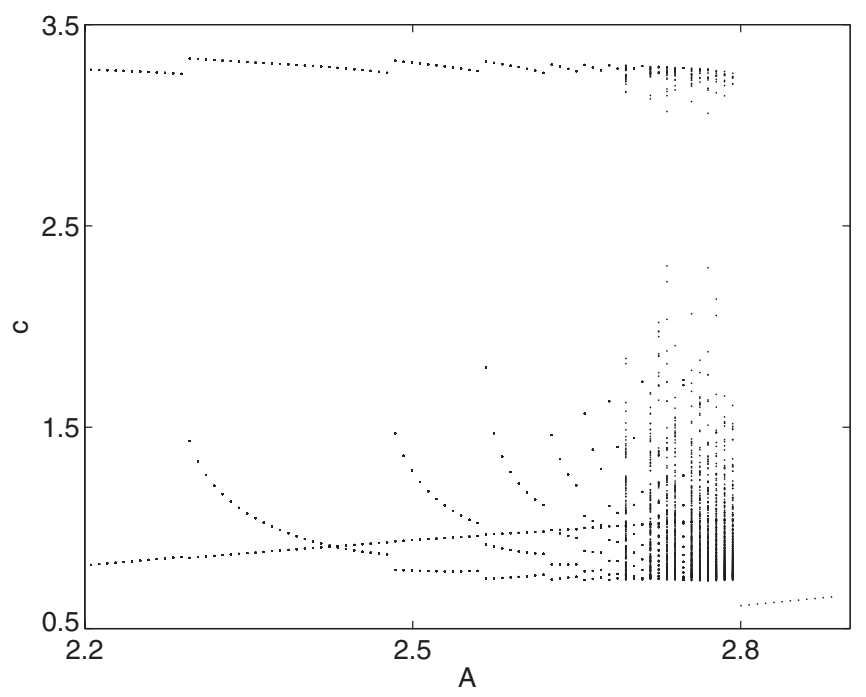

FIG. 6. Plot of $c$ versus $A$ taken from the Poincaré section, where $\epsilon=0.137, \bar{\epsilon}=8.00, a=4.17, b=0.85, \bar{A}=3.50$, and $A$ decreases. Observe the period-adding cascades of PQS stable self-pulsations, $\Pi^{(n)}$. The Hopf bifurcation occurs at $A \approx 2.8$, just to the right of where the chaotic motion ceases in the figure.

fixed $n$ the periodic self-pulsations $\Pi^{(n)}$ are organized along isolas $\mathcal{I}_{n}, n=3,4,5,6, \ldots$. Indeed, most of the qualitative information obtained by changing $\Delta \omega$ can be found from the previous case, when $A$ is the control parameter. For the sake of completeness, we give the aforementioned parameter dependence on $\Delta \omega[16]$ :

$$
\bar{A}(\Delta \omega)=\frac{\alpha}{1+\left(\frac{\Delta \omega}{\bar{\Gamma}}\right)^{2}}, \quad A(\Delta \omega)=\frac{\beta}{1+\left(\frac{\Delta \omega}{\Gamma}\right)^{2}},
$$
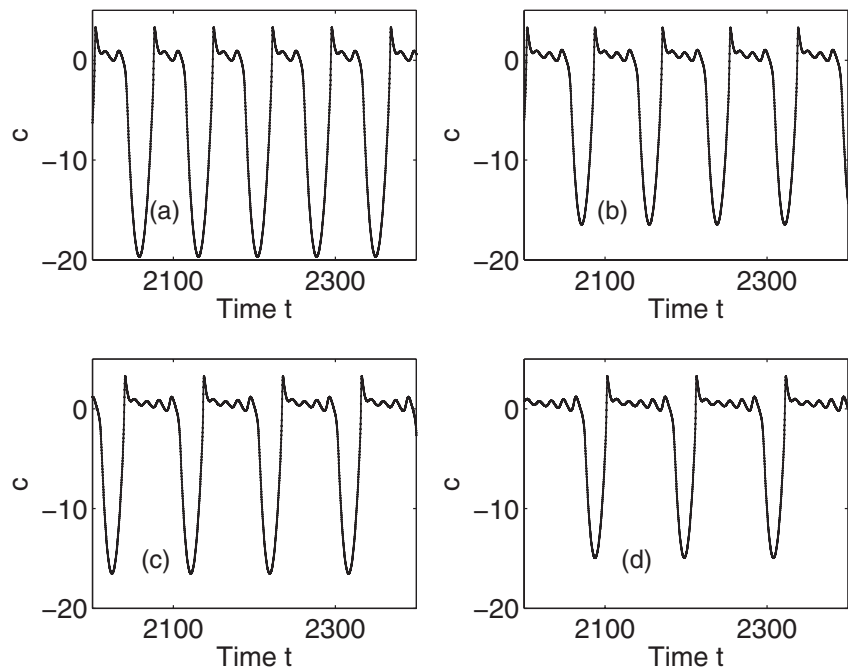

FIG. 7. Plot of $c$ versus time $t$, where $\epsilon=0.137, \bar{\epsilon}=8.00$, $a=4.17, b=0.85$, and $\bar{A}=3.50$. for the stable PQS self-pulsations, $\Pi^{(n)}, n=3,4,5,6$. The large negative value of $c$, down to less than -15 , corresponds to a very small value of $I$, where $I=e^{c}$, (a) $\Pi^{(3)}$ for $A=2.40$; (b) $\Pi^{(4)}$ for $A=2.55$; (c) $\Pi^{(5)}$ for $A=2.60$; (d) $\Pi^{(6)}$ for $A=2.65$. For each stable PQS self-pulsation, $\Pi^{(n)}$, we can see the reinjection (leading spike) and $n-1$ undulations (undamped relaxations).

$$
a(\Delta \omega)=\left(\frac{1+\left(\frac{\Delta \omega}{\Gamma}\right)^{2}}{1+\left(\frac{\Delta \omega}{\bar{\Gamma}}\right)^{2}}\right) a(0),
$$

where $\Gamma$ is an effective parameter.

\section{CONCLUSIONS AND DISCUSSION}

We have found that in the 3-2LM for the $\mathrm{CO}_{2} \mathrm{LSA}$ the periodic PQS self-pulsations $\Pi^{(n)}$ are organized along isolas $\mathcal{I}_{n}$, as either the incoherent pump parameter $A$ or the cavity frequency detuning $\Delta \omega$ is changed. This is a generic property of the model, and to our knowledge is reported for the first time for the 3-2LM. For a suitable value of the parameter $\bar{A}$, related to the population at thermal equilibrium in the absorber, the peculiar coexistence of periodic PQS stable self-pulsations induces the phenomenon known as period-adding cascades as either $A$ or $\Delta \omega$ is changed. As for the stability of the periodic PQS self-pulsations, a typical pattern emerges: any $\Pi^{(n)}$ is stable in an $A$ interval, or in a $\Delta \omega$ interval, bounded by period doubling and fold bifurcations. We have also shown that the size of this interval of stability becomes smaller with larger $n$, and that the isolas disappear one by one as the parameter $\bar{A}$ decreases.

We have implemented a method to construct the isolas $\mathcal{I}_{n}$ and to approximate the homoclinic connections from basic periodic orbits to themselves, as well as the homoclinic tangency. Along the isolas, splicing in an additional undulation in a solution near the right-hand fold of $\Pi^{(n)}$ provides an approximate starting solution near the right-hand fold of $\Pi^{(n+1)}$, which is then refined by a Newton iteration, and subsequently continued in order to trace out the entire isola $\mathcal{I}_{n}$. For large $n$ these solutions approximate homoclinic orbits, while at the fold on the right of the isolas they approximate a homoclinic tangency with increasing accuracy.

The presence of homoclinic orbits has been reported in previous studies within the context of the 3-2LM. Periodadding cascades of periodic orbits can be closely related to the presence of homoclinic tangencies, as shown by Gaspard and Wang [42], where a detailed numerical analysis shows how the existence of a tangent homoclinic orbit explains the sequence of MMOs and how symbolic dynamics with three symbols classifies the associated periodic windows of the MMOs. As a result of this work [42], any $\Pi^{(n)}$ can be identified with a fixed point of a map representing the flow in the vicinity of the homoclinic tangency [31]. A comprehensive study of these homoclinic bifurcation curves requires an extensive analysis of codimension-2 bifurcations, as carried out for the single-mode semiconductor laser subject to optical injection in Ref. [43]. In our system, these parameters could be either $(A, \bar{A})$ or $(\Delta \omega, \alpha)$. It would also be of interest to implement control techniques in nonlinear dynamics [44] in the context of $\mathrm{a} \mathrm{CO}_{2}$ LSA experiment. This would allow finding and tracking stable and unstable $\Pi^{(n)}$, and as a result confirm the existence of isolas $\mathcal{I}_{n}$ in this versatile experimental system.

To conclude, we highlight some relevant differences between a molecular model (MM) for the LSA [39] and the 3-2LM discussed here. First, as pointed out above, the 3-2LM assumes that the amplifier in the LSA is a three-level system, while in the MM the amplifier is described by a refined fourlevel system $[39,40]$ that provides reasonable agreement with 
experimental results. The latter description of the amplifier also explains experimental results in other types of $\mathrm{CO}_{2}$ lasers $[37,45]$. Since the features of the MM are consistent with previous $\mathrm{CO}_{2}$ laser models, it is appropriate to compare them to those of the 3-2LM, which already gives surprisingly good agreement with experimental observations [9,10,13]. Also, the 3-2LM formally arises as a limiting case of the MM for large vibrational-rotational relaxation rates. However, the MM parameters do not always allow such a simplification, which could result in wrong predictions. We also note that the PQS self-pulsations are stable in a pump interval bounded by period doubling bifurcations along the isolas, while in the 3-2LM these bounds are a fold and a period doubling. Furthermore, in the 3-2LM the origin of the isolas is the "isola formation point mechanism" [46], as we change $(A, \bar{A})$. In the MM the situation is somewhat more complicated [39]: there the loci of folds do not always have the form of a lobe; in fact they may contain more than one minimum with respect to $\bar{A}$ in the two-parameter $(A, \bar{A})$ diagram. In this scenario the isola first breaks into two separate isolas, which then disappear one after the other. Nevertheless, apart from these and several other differences in detail, the coarse grain bifurcation picture is qualitatively similar in both models. In light of these bifurcation structures it is of interest to use the MM in other physical settings of experimental interest, such as the study of chaotic dynamics and the onset of chaotic synchronization in coupled LSA, both of which have been considered experimentally.

\section{ACKNOWLEDGMENTS}

This work was supported by NSERC (Canada), the Instituto de Física and VIEP at BUAP (Mexico), and CONACYT (Mexico).
[1] F. T. Arecchi, in Instabilities and Chaos in Quantum Optics, edited by F. T. Arecchi and R. G. Harrison (Springer-Verlag, Berlin, 1987), p. 9.

[2] P. Mandel, Theoretical Problems in Cavity Nonlinear Optics (Cambridge University Press, New York, 1997); paperback edition 2005.

[3] S. Wieczorek, B. Krauskopf, T. B. Simpson, and D. Lenstra, Phys. Rep. 416, 1 (2005).

[4] O. R. Wood and S. E. Shwarz, Appl. Phys. Lett. 11, 88 (1967).

[5] N. B. Abraham, P. Mandel, and L. M. Narducci, in Progress in Optics $X X V$, edited by E. Wolf (North-Holland, Amsterdam, 1988), p. 1.

[6] I. Burak, P. Houston, D. Sutton, and J. Steinfeld, IEEE J. Quantum Electron. 7, 73 (1971).

[7] J. Dupré, F. Meyer, and C. Meyer, Rev. Phys. Appl. (Paris) 10, 285 (1975).

[8] E. Arimondo, F. Casagrande, L. Lugiato, and P. Glorieux, Appl. Phys. B: Photophys. Laser Chem. 30, 57 (1983); E. Arimondo and E. Menchi, ibid. 37, 55 (1985).

[9] M. Tachikawa, K. Tanii, M. Kajita, and T. Shimizu, Appl. Phys. B: Photophys. Laser Chem. 39, 83 (1986); M. Tachikawa, K. Tanii, and T. Shimizu, J. Opt. Soc. Am. B 4, 387 (1987).

[10] M. Tachikawa, F. L. Hong, K. Tanii, and T. Shimizu, Phys. Rev. Lett. 60, 2266 (1988); M. Tachikawa, K. Tanii, and T. Shimizu, J. Opt. Soc. Am. B 5, 1077 (1988).

[11] D. Hennequin, F. de Tomasi, B. Zambon, and E. Arimondo, Phys. Rev. A 37, 2243 (1988).

[12] D. Dangoisse, A. Bekkali, F. Pappof, and P. Glorieux, Europhys. Lett. 6, 335 (1988).

[13] M. Lefranc, D. Hennequin, and D. Dangoisse, J. Opt. Soc. Am. B 8, 239 (1991).

[14] V. V. Nevdakh, O. L. Gaiko, and L. N. Orlov, Opt. Commun. 127, 303 (1996).

[15] L. de B. Oliveira-Neto, G. J. F. T. da Silva, A. Z. Khoury, and J. R. Rios-Leite, Phys. Rev. A 54, 3405 (1996); P. C. de Oliveira, M. B. Danailov, Y. Liu, and J. R. Rios-Leite, ibid. 55, 2463 (1997).
[16] H. L. D. de S. Cavalcante and J. R. Rios Leite, Chaos 18, 023107 (2008).

[17] F. Fernández-Sánchez, E. Freire, and A. J. Rodríguez-Luis, Dyn. Stab. Syst. 12, 319 (1997).

[18] R. J. Olsen and D. G. Vlachos, J. Phys. Chem. A 103, 7990 (1999).

[19] E. J. Doedel, in Proceedings of Special Year in Energy Mathematics, University of Wyoming, edited by K. I. Gross (SIAM, Laramie, 1984), pp. 103-138.

[20] T. Tohei, M. Tachikawa, and T. Shimizu, Phys. Rev. A 45, 5166 (1992).

[21] M. K. Stephen Yeung and S. H. Strogatz, Phys. Rev. E 58, 4421 (1998).

[22] C. Bonatto and J. A. C. Gallas, Phys. Rev. E 75, 055204(R) (2007).

[23] B. Krauskopf and S. Wieczorek, Physica D 173, 97 (2002).

[24] C. Mayol, M. A. Natiello, and M. G. Zimmermann, Int. J. Bifurcation Chaos 11, 2587 (2001).

[25] W. Lauterborn and I. Eick, J. Opt. Soc. Am. B 5, 1089 (1988).

[26] J. L. A. Dubbeldam, B. Krauskopf, and D. Lenstra, Phys. Rev. E 60, 6580 (1999); A. Huber and P. Szmolyan, SIAM J. Appl. Dyn. Syst. 4, 607 (2005)

[27] T. Erneux, P. Peterson, and A. Gavrielides, Eur. Phys. J. D 10, 423 (2000); G. Kozyreff and T. Erneux, Eur. J. Appl. Math. 14, 407 (2003).

[28] M. Brons, T. J. Kaper, and H. G. Rotstein, Chaos 18, 015101 (2008); M. Desroches, B. Krauskopf, and H. M. Osinga, ibid. 18, 015107 (2008); N. Baba and K. Krisher, ibid. 18, 015103 (2008); M. Higuera, J. Porter, and E. Knobloch, ibid. 18, 015104 (2008).

[29] E. J. Doedel, B. E. Oldeman, A. R. Champneys, F. Dercole, T. F. Fairgrieve, Y. Kuznetsov, R. C. Paffenroth, B. Sandstede, X. J. Wang, and C. H. Zhang, AUTO-07p, Version 0.7: Continuation and Bifurcation Software for Ordinary Differential Equations (Concordia University, Montréal, Canada, 2010).

[30] P. Alcantara Jr. et al., J. Opt. Soc. Am. B 12, 1326 (1995).

[31] B. Zambon, Phys. Rev. A 44, 688 (1991). 
[32] A. R. Zeni, J. A. C. Gallas, A. Fioretti, F. Papoff, B. Zambon, and E. Arimondo, Phys. Lett. A 172, 247 (1993).

[33] F. Papoff, A. Fioretti, and E. Arimondo, Phys. Rev. A 44, 4639 (1991).

[34] A. Algaba, M. Merino, and A. J. Rodríguez-Luis, Int. J. Bifurcation Chaos 11, 513 (2001).

[35] P. Hirschberg and C. Laing, Physica D 89, 1 (1995).

[36] A. R. Champneys and A. J. Rodriguez-Luis, Physica D 128, 130 (1999).

[37] C. L. Pando L., R. Meucci, M. Ciofini, and F. T. Arecchi, Chaos 3, 279 (1993).

[38] F. Papoff, A. Fioretti, E. Arimondo, G. B. Mindlin, H. Solari, and R. Gilmore, Phys. Rev. Lett. 68, 1128 (1992).

[39] E. J. Doedel, B. E. Oldeman, C. L. Pando L., Int. J. Bifurcation Chaos 21, 305 (2011).

[40] C. L. Pando L., Phys. Lett. A 210, 391 (1996).
[41] Y. Liu, L. C. Barbosa, and J. R. Rios-Leite, Phys. Lett. A 193, 259 (1994).

[42] P. Gaspard and X.-J. Wang, J. Stat. Phys. 48, 151 (1987).

[43] S. Wieczorek and B. Krauskopf, Nonlinearity 18, 1095 (2005); S. Wieczorek, B. Krauskopf, and D. Lenstra, Phys. Rev. Lett. 88, 063901 (2002); S. Wieczorek and B. Krauskopf, Opt. Commun. 172, 279 (1999).

[44] S. Boccaletti, C. Grebogi, Y. C. Lai, H. Mancini, and D. Maza, Phys. Rep. 329, 103 (2000); S. Schikora, P. Hövel, H.-J. Wünsche, E. Schöll, and F. Henneberger, Phys. Rev. Lett. 97, 213902 (2006); J. Sieber, A. Gonzalez-Buelga, S. A. Neild, D. J. Wagg, and B. Krauskopf, ibid. 100, 244101 (2008).

[45] R. Meucci, M. Ciofini, and P. Y. Wang, Opt. Commun. 91, 444 (1992); A. Varone, A. Politi, and M. Ciofini, Phys. Rev. A 52, 3176 (1995).

[46] D. Dellwo, H. B. Keller, B. J. Matkowski, and E. L. Reiss, SIAM J. Appl. Math. 42, 956 (1982). 